



\section{REFLEXIONES EN TORNO A LA REPROBACIÓN ESTUDIANTIL, DESIGUALDAD SOCIAL Y EQUIDAD DE GÉNERO EN ESTUDIANTES DE LICENCIATURA.*}

\author{
Blanca Noemí Silva Gutiérrez \\ Doctora en Ciencias \\ Universidad de Guadalajara - CUCEA \\ bnoemisg@gmail.com
}

\author{
Amada Lydia Rodríguez Téllez \\ Doctora en Educación Superior \\ Universidad de Guadalajara - CUCEA \\ amadalydia7@gmail.com
}

\author{
Raúl Vicente Flores \\ Doctor en Ciencias del Desarrollo Humano \\ Universidad de Guadalajara - CUCEA \\ raulvi59@gmail.com
}

\author{
Miguel Antonio Ruiz de Alba \\ Licenciado en Sistemas de Información. Maestrante en Educación. \\ Área Investigación Educativa y Docencia \\ Universidad de Guadalajara - CUCEA \\ maruiz@redudg.udg.mx
}

\section{RESUMEN}

El presente trabajo es parte de una investigación realizada en la Universidad de Guadalajara durante el período 2009-2012. Tiene por objetivo analizar desde un enfoque integral, los aspectos que desde la perspectiva de los estudiantes inciden en la reprobación estudiantil de las licenciaturas del Centro Universitario de Ciencias Económico Administrativas -CUCEA-, que se encuentran en estatus irregular por aplicación de los artículos 33, 34 y 35 de la normatividad universitaria. Interesa destacar que la reprobación es una manifestación de los problemas sociales provocados por la desigualdad social, porque a pesar de que los reprobados tuvieron la oportunidad de ingresar a la universidad después de un proceso de selección altamente competitivo, como estudiantes universitarios enfrentan situaciones derivadas del contexto económico social e institucional que los llevan a vivir la posible exclusión de la carrera que cursan a partir de los rígidos criterios institucionales para la permanencia en las aulas y la falta de apoyos o programas efectivos para este grupo vulnerable. También interesa analizar desde un acercamiento a la perspectiva de género, el desempeño académico de las y los universitarios, que muestra indicadores positivos en las mujeres, tanto en términos de matrícula mayor que la de los varones (56.6\%) y en los índices de reprobación menor (42\%). En este documento se analizan los factores de reprobación de las y los estudiantes y se plantean propuestas que les permitan avanzar en el proceso de educativo.

Palabras clave: estudiantes universitarios, reprobación, desigualdad social, desigualdad educativa y equidad de género.

\begin{abstract}
The present study is part of a research initiative conducted at the University of Guadalajara during the period of 2009-2012. Its objective is to take a comprehensive approach to the analysis of factors which, from the perspective of students enrolled, impact academic failure among Bachelor's degree candidates at the Centro Universitario de Ciencias Económico Administrativas-CUCEA-who find themselves under irregular status by virtue of articles 33, 34, and 35 of University Standards and Regulations. It is worth noting that student failure is a manifestation of the problems induced by social inequality-although failed students were once granted the opportunity to attend the university as a result of a highly competitive selection process, as matriculated students they face situations rooted in a socio-economical and institutional context that cause them to experience a possible expulsion from the major of their choosing due to rigid institutional criteria for classroom attendance as well as a lack of support and effective programs for the vulnerable group in question. It is also worth analyzing academic achievement from a gender perspective, as student performance exhibits positive indicators among women in terms of a higher matriculation than men (56.6\%) as well as a lower index of failure (42\%). This article provides an analysis of the factors that incite failure among students and outlines proposals that will allow their advancement in the educational process.
\end{abstract}

Key words: college students, failure, social inequality, educational inequality and gender equity. 


\section{Introducción}

El presente documento tiene como objetivo analizar la reprobación estudiantil como manifestación de los problemas relacionados con las condiciones de desigualdad social que viven los estudiantes porque en su mayoría son estas las que dificultan el recorrido exitoso de su proceso educativo.

Pretende también reflexionar acerca de un hecho que parece mostrar avances en el proceso de empoderamiento de las mujeres: el aumento de la matrícula femenina en los estudios universitarios $-56.6 \%$ - respecto a los varones, así como menores índices de reprobación -42\%-, según datos de 2011. (Universidad de Guadalajara: 2011). Se considera que esta situación puede abonar a la construcción de la equidad de género. El concepto de género se entiende "como el conjunto de relaciones sociales que, con base en las características biológicas regula, establece y reproduce las diferencias entre hombres y mujeres. Se trata de una construcción social, de un conjunto de relaciones con intensidades específicas en tiempos y espacios diversos". (Ramos Escandón, 1992:12) siempre y cuando los modelos patriarcales y sus pautas culturales no sigan reproduciéndose.

El trabajo no se ubica rigurosamente dentro de la perspectiva de género porque según la clasificación de Nuñez Noriega (2007) tiene las restricciones propias de aquellos que se incluyen en la categoría de estudios de fenómenos sociales según diferencial por sexo, puesto que comparamos y analizamos datos provenientes de archivos oficiales; también la limitación de los estudios sobre las concepciones, actitudes y valoraciones de los sujetos, dado que también se construyen datos a partir de una encuesta y entrevistas semi-estructuradas, que nos proporcionaron información a partir de la opinión de los estudiantes; sin embargo, como Nuñez Noriega afirma, es un punto de partida útil para futuras investigaciones en las que se profundice sobre el tema.

\section{La reprobación en las licenciaturas del CUCEA ${ }^{1}$}

La investigación realizada destaca la importancia de considerar la reprobación como una manifestación de los problemas sociales provocados por la desigualdad social, porque a pesar de que los reprobados tuvieron la oportunidad de ingresar a la universidad después de un proceso de selección altamente competitivo, como estudiantes universitarios enfrentan situaciones derivadas del contexto económico, social e institucional que los llevan a vivir la posible exclusión de la carrera que cursan a partir de la normatividad que establece criterios rígidos para la permanencia en las aulas, sin ofrecer apoyos o programas efectivos para este grupo vulnerable. En términos generales no se aprecia la aplicación de políticas para la construcción de la equidad social ni para la equidad de género ya que en el ámbito laboral-profesional se reproducen los papeles tradicionales que asignan preferencia a los varones en cuanto al acceso a puestos y salarios superiores, independientemente de que en términos generales el desempeño académico de las mujeres universitarias muestra indicadores positivos.

Sin embargo, aún en las condiciones de desigualdad antes mencionadas, Ilama la atención que durante el periodo estudiado, en las carreras del área económico administrativa la matrícula de población femenina alcanza el $56.6 \%$, lo que indica que en el proceso de selección presentaron mejores resultados que sus compañeros varones. Además, el porcentaje de reprobación femenino es menor que el masculino. Del total de los 14 mil 602 estudiantes de las 10 licenciaturas de este centro universitario, en el ciclo escolar en el que se realizó la encuesta, el número de reprobados a los que se aplicaron los artículos 33, 34 y 35 corresponde

1 Las licenciaturas analizadas son: Administración, Administración Financiera y Sistemas, Administración Gubernamental y Políticas Públicas, Contaduría Pública, Economía, Mercadotecnia, Negocios Internacionales, Recursos Humanos, Sistemas de Información y Turismo; la reprobación y estatus irregular en los artículos 33, 34 y 35 se localiza principalmente en las carreras de Mercadotecnia, Negocios Internacionales, Administración, Turismo y Economía. 
a un mil quinientos dieciocho personas, es decir, el 10.3\% del estudiantado. Situación similar se encuentra en los ciclos de años posteriores. De este total (incluidos los tres artículos) el $42 \%$ corresponde a mujeres y el $58 \%$ a hombres, no obstante el análisis por cada artículo muestra cifras diferenciadas.

\section{La dimensión del problema en el CUCEA}

Las cifras de reprobación no son menores en términos de cantidad de personas que en los semestres de 2010 a 2012 estaban en situación de riesgo, ya que el artículo 33 se aplica al estudiante que por cualquier circunstancia no logre una calificación aprobatoria en el periodo extraordinario, de modo que deberá repetir la materia en el ciclo escolar inmediato siguiente en que se ofrezca, teniendo la oportunidad de acreditarla durante el proceso de evaluación ordinario o en el periodo extraordinario. En caso de que el estudiante no logre acreditar la asignatura en los términos de este artículo, será dado de baja (REGEPE:2000). Los criterios son más rígidos en los artículos 34 y 35.

La magnitud de la cantidad de jóvenes que padecen esta situación nos condujo a reflexionar acerca de la necesidad de analizar la problemática como un fenómeno complejo en el que están presentes elementos del contexto social, institucional e individual. Aunque estos estudiantes ingresaron a la universidad, varios factores les impiden continuar satisfactoriamente sus estudios; a la "aparente igualdad de oportunidades se suma la desigualdad de condiciones objetivas" (Bordieu y Passeron: 1998) para terminar exitosamente el proceso educativo. Ante esta situación surge la pregunta: ¿Cuáles son los factores que inciden en la reprobación de los estudiantes del CUCEA?

\section{Aspectos Metodológicos}

Se partió de la construcción de la base de datos registrados en el Sistema Integral de Información y Administración Universitaria (SIIAU)

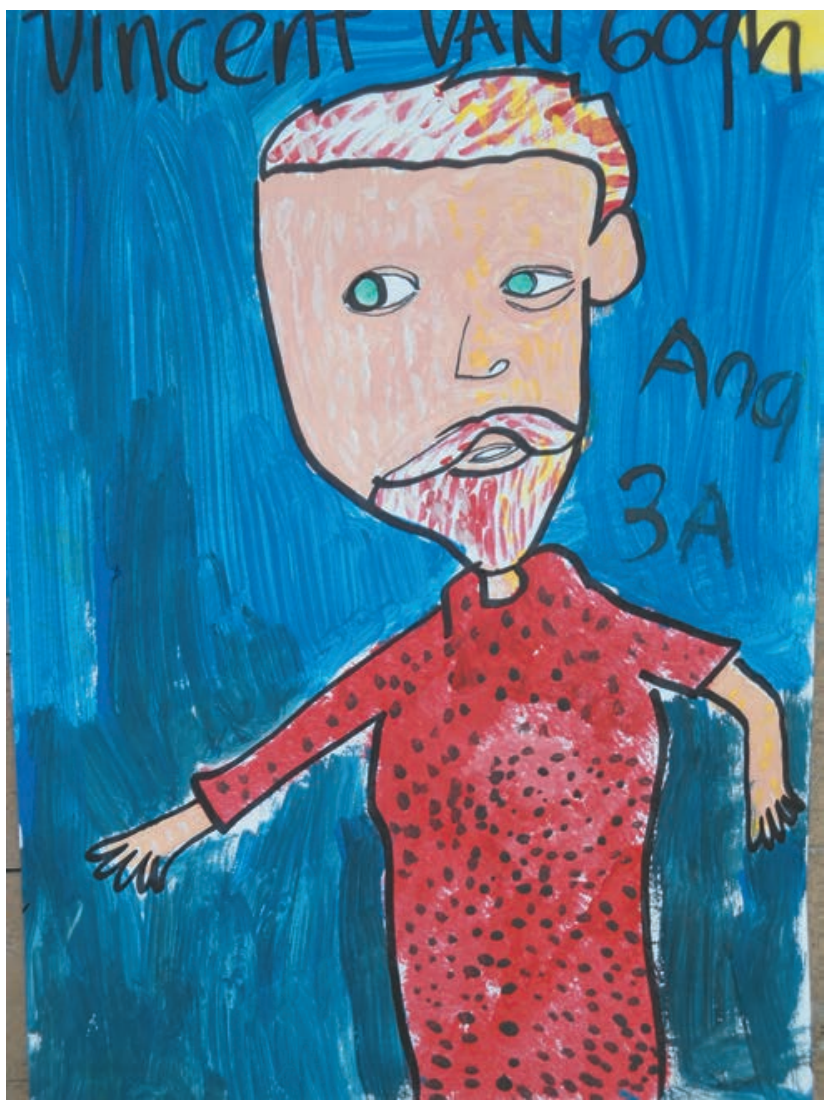

Imagen fotografía María Isabel Jasbón

para identificar los casos de los estudiantes que se encontraron en los artículos antes mencionados durante el calendario escolar 2009 B y que continuaron estudiando en los siguientes calendarios.

En este documento se estudia la situación de reprobación a partir de la opinión de los alumnos y la identificación de los principales factores involucrados en la condición de estatus irregular por aplicación de los artículos 33, 34 y 35 del REGEPE. Así mismo, se analiza esta problemática a partir del concepto de desigualdad social.

El estudio se enfocó a analizar los diferentes factores que inciden en la reprobación en el nivel de educación superior como parte de una problemática multicausal y compleja relacionada con conceptos como repitencia, rezago, deserción, baja o abandono y en última instancia con el fracaso escolar. Alrededor de estos conceptos las investigaciones realizadas 
han señalado motivos internos y externos a las instituciones educativas, constituyendo en su abordaje una doble vertiente educativa y social debido a que además de influir negativamente en la formación de los estudiantes, la situación de fracaso también afecta las posibilidades de empleo y promoción personal y profesional que acrecienta las probabilidades de marginación y diversos problemas sociales. El análisis desde la perspectiva de género, inicialmente no contemplada en este estudio, resulta ser un factor que aporta visibilidad a problemas inadvertidos fuera de este razonamiento.

La reprobación se estableció con el número total o porcentaje de estudiantes que de acuerdo con el Reglamento General de Evaluación y Promoción de Estudiantes de la Universidad de Guadalajara (REGEPE) no cumplió con alguno de los requisitos de evaluación formulados en el programa de estudios de las distintas asignaturas que integran el plan curricular de las licenciaturas del CUCEA y que impiden cursar nuevas materias o permanecer en la institución.

La condición estudiantil de estatus irregular por los artículos antes mencionados se vincula al concepto presentado en diversos estudios como "repitencia", término definido como la acción de cursar repetidamente una actividad académica, sea por mal rendimiento del estudiante o por causas ajenas al ámbito académico. Según el modelo curricular, ésta se puede presentar en la educación superior de distintas maneras; puede estar referida a todas las actividades académicas de un período determinado (año, semestre o trimestre), o bien, a cada asignatura.

Los resultados que se presentan más adelante corresponden primero a la fase de investigación cuantitativa que consistió en un estudio descriptivo y transversal dirigido a la población estudiantil (un mil quinientos dieciocho alumnos) que se encontraba en estatus irregular por reprobación en el calendario 2009B.

La población objeto de estudio corresponde al total de estudiantes de licenciatura ubicados en alguno de los artículos antes señalados registrados en el SIIAU. A partir de la información contenida en este sistema fue posible identificar y localizar a los estudiantes en los horarios de clases para pasar a la fase de investigación cualitativa, solicitar su participación en la encuesta y programar la realización de las entrevistas.

Para los estudiantes localizados en los artículos 33 y 34, quienes representan la proporción más alta entre los estudiantes que viven este problema, (SIIAU 2009-2010), se diseñó una muestra representativa estratificada por carrera. En el artículo 33 de un total de 896 estudiantes se obtuvo una muestra de 271 participantes. Para el 34 de un total de 548 alumnos la muestra consistió en 225 sujetos.

En lo correspondiente al artículo $35^{2}$, en el trabajo de campo se intentó realizar un censo (el número de estudiantes en esa condición es menor, sin embargo, corresponde a la situación de extremo riesgo de baja de la institución). En esta población la principal dificultad para incluirlos en el estudio se derivó de la alta irregularidad en cuanto a la asistencia a clases, a pesar de encontrarse en situación especial. De acuerdo con lo anterior, de un total de 74 alumnos se logró la participación de 46 personas de ambos sexos que corresponde al $62 \%$.

El trabajo de campo significó un esfuerzo y dedicación muy importantes, particularmente por la dificultad de localizar a los estudiantes, especialmente debido a que uno de los aspectos que caracterizan a este segmento estudiantil es precisamente la constante inasistencia a clases. Para atender esta problemática, se contó con la colaboración de estudiantes de servicio social, quienes acudían a las aulas para localizar a los integrantes de la muestra y aplicarles la encuesta o en su caso, solicitar

2 Los estudiantes del artículo 35 en estatus activo corresponden a los casos que se les aplicó el criterio de expulsión de la Institución pero que a partir de procedimientos especiales y el acuerdo ante el Consejo del Centro Universitario, se autorizó otorgarles la oportunidad de concluir la carrera, bajo requerimientos especiales como contar con un tutor y regularizar la (s) materia (s) que adeudan. 
que se presentaran fuera del horario de clases en el área en donde podían aplicar el cuestionario.

Por las características anteriores, el trabajo de campo se prolongó por un semestre completo, durante el que se buscó a algunos estudiantes reiteradamente y solo cuando se excedía a más de tres visitas y no se obtenía respuesta favorable, se sustituían por otra persona de la misma carrera y con el mismo estatus, respecto a la situación de estar ubicados en el SIIIAU en determinado artículo. A esto se agrega también la dificultad de contactar a las jóvenes porque cuando un investigador hablaba por teléfono a su casa con el fin de concertar una cita para la entrevista y éstos no se encontraban, los padres o hermanos mostraban desconfianza e interrogaban al profesor.

El total de participantes en la encuesta fue de 542 jóvenes de ambos sexos, de los distintos turnos y carreras, que representan el $35.70 \%$ de los estudiantes de los artículos 33, 34 y 35 del ciclo escolar respectivo.

Se diseñó un cuestionario que incluye los siguientes aspectos: datos socio-demográficos, información académica del estudiante -procedencia, ingreso y trayectoria escolar-, opinión del estudiante acerca de los motivos que los han llevado a la reprobación -sociales y familiares, económicos, psicológicos, relativos al rendimiento escolar, físicos, atribuibles al profesor- y valoración de los servicios que ofrece a los estudiantes la institución. El cuestionario se diseñó a partir de la revisión y análisis de información procedente de diversos estudios relacionados con el propósito de la investigación. Se aplicó la prueba piloto respectiva y se sometió a criterios de validación a través de expertos. El cuestionario se aplicó a los estudiantes de manera individual mediante entrevistas.

El trabajo de campo recupera la opinión de los estudiantes acerca de los aspectos sociales, institucionales y personales que desde su expe- riencia están presentes y constituyen los motivos principales de reprobación.

\section{Análisis de resultados}

\section{Reprobación y estatus irregular por artícu- los 33, 34 y 35 en las carreras del CUCEA}

La feminización de la matrícula en el CUCEA así como en otras instituciones de educación superior es un hecho insoslayable, de 2009 a la fecha, según el informe de Actividades 20102011 del rector de este centro, Mtro. Tonatiuh Bravo Padilla, la matrícula se incrementó en 5.52 por ciento, alcanzando un total de 15 mil 033 estudiantes de los cuales el 55.9 por ciento son mujeres. (UdeG: 2011). En 2012 el porcentaje de población femenina comprendió el $56.5 \%$.

Si bien esta es una noticia que refleja de alguna manera avances en el proceso de empoderamiento de las mujeres durante su formación académica, el buen desempeño de las jóvenes no significa que luego como profesionistas tengan garantizada una mejor situación laboral o acceso a puestos directivos.

Estudiosas del tema como Rosaura Ruiz (2005), señalan que "permanece la falta de congruencia entre los altos índices de egresadas femeninas y las oportunidades de empleo, entre la preparación académica y las diferencias salariales entre hombres y mujeres" debido a barreras no visibles, que dificultan su ascenso.

No obstante, es posible encontrar situaciones en que las mujeres alcanzan puestos directivos pero siguen reproduciendo esquemas patriarcales con conductas autoritarias o de mujeres que ocupan el puesto pero se limitan a obedecer los designios de sus superiores en la escala jerárquica de mando.

En las siguientes Tablas se presentan los datos de los estudiantes reprobados según la ubicación en cada artículo y sexo al que pertenece 


\begin{tabular}{|l|c|c|}
\hline \multicolumn{3}{|c|}{ Sexo } \\
\hline \multicolumn{3}{|c|}{ Tabla 1 Art. 33 $(\mathrm{n}=271)$} \\
\hline & Frecuencia & Porcentaje \\
\hline Masculino & 135 & 49.8 \\
\hline Femenino & 136 & 50.2 \\
\hline Total & 271 & 100 \\
\hline
\end{tabular}

Fuente: Encuesta sobre Motivos de Reprobación, CUCEA 2010

\begin{tabular}{|l|c|c|}
\hline \multicolumn{3}{|c|}{ Sexo } \\
\hline \multicolumn{3}{|c|}{ Tabla 1 Art.34 $(\mathrm{n}=225)$} \\
\hline & Frecuencia & Porcentaje \\
\hline Masculino & 123 & 54.7 \\
\hline Femenino & 102 & 45.3 \\
\hline Total & 225 & 100.0 \\
\hline
\end{tabular}

Fuente: Encuesta sobre Motivos de Reprobación, CUCEA 2010

\begin{tabular}{|l|c|c|}
\hline \multicolumn{3}{|c|}{ Sexo } \\
\hline \multicolumn{3}{|c|}{ Tabla 1 Art. 35 $(\mathrm{n}=46)$} \\
\hline & Frecuencia & Porcentaje \\
\hline Masculino & 26 & 56.5 \\
\hline Femenino & 20 & 43.5 \\
\hline Total & 46 & 100 \\
\hline
\end{tabular}

Fuente: Encuesta sobre Motivos de Reprobación, CUCEA 2010

Los datos muestran en el artículo 33, un porcentaje ligeramente mayor de mujeres reprobadas que de hombres, pero al observar las Tablas de los artículos siguientes, se marcan las diferencias a favor de las mujeres, lo que indica que en general éstas superaron la situación de reprobación más favorablemente que los varones al no pasar al artículo siguiente.

En lo relativo a las materias principales que no aprueban los estudiantes y que los llevan al estatus irregular destacan preponderantemente matemáticas II (22.50\%) y matemáticas I (18.45\%). En tercer lugar se encuentra estadística I (6.27\%), seguida por economía II (3.87\%) y economía I (3.13\%), estadística II (2.95\%) y matemáticas IV (2.21\%), entre las principales.

Con las cifras anteriores se pude apreciar que la reprobación se concentra en un 59.4\% en materias del área cuantitativa, lo que revela una doble problemática, la deficiencia proveniente en esta área desde niveles de estudios previos, y la necesidad de establecer medidas efectivas de apoyo para las y los estudiantes porque queda claro que los cursos propedéuticos y las asesorías que ofrece el departamento a cargo de estos cursos, no han sido suficientes para atender esta situación. En estas asignaturas las mujeres tienden a reprobar más que los hombres, por ejemplo:

En artículo 33, Matemáticas I, el 47.5\% corresponde a hombres y el $52.4 \%$ a mujeres, en Matemáticas II, $35.5 \%$ a hombres y $64.4 \%$ a mujeres. En estadística I la situación cambia pues el $66.6 \%$ son hombres y 33.3 mujeres.

En artículo 34, en Matemáticas I, 47\% hombres y $52.9 \%$ mujeres, por el contrario en Matemáticas II, $56.6 \%$ hombres y $43.3 \%$ mujeres. En Estadística I, $45.4 \%$ hombres y $54.5 \%$ mujeres.

En artículo 35, tanto en matemáticas I como II, no se reportó reprobación entre las mujeres y únicamente dos jóvenes estaban en esta situación.

La revisión del resto de asignaturas principales en que los estudiantes no alcanzan la calificación aprobatoria, indica que no existe una marcada tendencia con sexo al respecto.

\section{Perfil de los estudiantes}

En los siguientes puntos queremos hacer notar que el problema de la desigualdad social más que la diferencia por sexo, se manifiesta en los resultados obtenidos en las diferentes áreas consideradas en este estudio; especialmente que el origen socioeconómico de las y los estudiantes está asociado con el mayor o menor logro en su quehacer educativo, porque a pesar de que "las probabilidades de acceso a la enseñanza han crecido para todas las categorías sociales" (Bourdieu y Passeron $1998,280)$ no ocurre lo mismo con las condiciones de permanencia. 


\section{Trabajo}

Es necesario destacar que el $72.5 \%$ del alumnado tiene trabajo remunerado y este porcentaje es mayor ( $85 \%$ ) en el grupo que se encuentran en el artículo 35, o sea, se encuentran en situación extrema respecto al riesgo de baja o expulsión de la carrera. De esta población $58.9 \%$ son hombres y $41 \%$ mujeres. Únicamente $15 \%$ no cuenta con empleo, distribuidos de manera similar entre los dos sexos.

Existen estudiantes que ya pasaron por este proceso y actualmente están cursando una nueva carrera, aunque subsiste la posibilidad de encontrar casos en los que la Comisión de Educación y el Consejo de Centro, a partir de un análisis cuidadoso, otorgan la posibilidad de continuar y terminar la carrera bajo condiciones específicas.

La proporción de jóvenes que trabajan es alta también entre quienes se encuentran en los otros artículos. El 73\% ubicado en artículo 34 cuentan con empleo remunerado y el $70 \%$ en el artículo 33. La distribución por sexo es similar, en el artículo 33 se encuentra el $55.2 \%$ de hombres y $44.7 \%$ mujeres; $y$ en el art. 34 , las cifras muestran el $56 \%$ para los hombres y el 43,9 $\%$ para las mujeres.

El tiempo que los estudiantes dedican al trabajo es variable pero representa una proporción importante en las actividades cotidianas, a las que se suman los tiempos de traslado y los horarios escolares. Los datos generales muestran los porcentajes respecto a las horas dedicadas diariamente. Entre quienes laboran de 4 a 5.5 horas diarias se reportó el $13.4 \%$, de 6 a 7.5 horas representa el $23.9 \%$, de 8 a 9.5 horas el $16.9 \%$ y de 10 a 13 horas el $4.1 \%$. En términos generales el $40.8 \%$ de los participantes trabajan entre 6 y 9.5 horas diarias. La proporción porcentual entre ambos sexos es parecida.

Hay que hacer notar la desventaja de contar o no con automóvil. De los ubicados en el artículo 33 , la mayoría -173 alumnos- utiliza trans- porte público, de los 81 jóvenes que cuentan con automóvil para trasladarse $67 \%$ son hombres y mujeres solo $32 \%$; en el artículo 34 se contabiliza $63 \%$ de hombres y $36.7 \%$ mujeres; y en el artículo $35,68 \%$ hombres y $32 \%$. mujeres. El resto vive cerca y llega caminando, en ocasiones viaja en transporte público o en vehículo particular de otra persona.

Lógicamente la situación laboral más el tiempo utilizado en transportarse, incide en la cantidad de horas que dedican a las actividades académicas aún cuando la mayoría (99.6\%) tenga acceso al servicio de Internet, ya sea en el hogar, fuera de él o de forma conjunta. De manera particular, en cuanto al tiempo que dedican para realizar actividades académicas mediante esta herramienta, se reportaron los siguientes porcentajes: el $34.8 \%$ dedica dos horas, el $20.4 \%$ dedican por lo menos una hora y el $18.8 \%$ dedican tres horas en promedio. En todos los artículos 33, 34 y 35, las diferencias entre ambos sexos es de minutos. Se aprecia que en cualquier caso, el tiempo dedicado a la actividad de aprendizaje es reducida para efectos de obtener el conocimiento necesario para ser profesionista de una carrera universitaria. Hay que recordar también que la diversión y el entretenimiento no son sacrificados por la mayoría de estos jóvenes, cuyas edades oscilan entre los 18 y 26 años de edad.

A la pregunta sobre el motivo principal para estar trabajando, las respuestas en orden de importancia fueron las siguientes: ser el proveedor principal de la familia (aún cuando un poco más del $90 \%$ son solteros), alcanza en la población masculina un porcentaje de $56.6 \%$ y en la femenina $43.3 \%$. Le sigue el de solventar gastos personales con $48.6 \%$ para los jóvenes y $51.3 \%$ para las jóvenes; seguido por la necesidad de contribuir al gasto familiar con $58.6 \%$ para hombres y 41.3 las mujeres.

En las entrevistas, los hombres manifestaron que el trabajo era la razón principal para reprobar mientras que para las mujeres, además del 


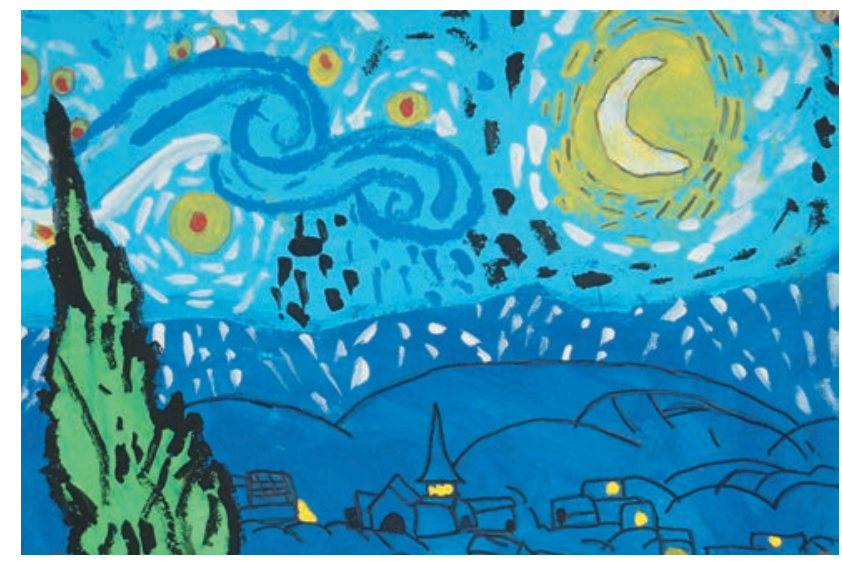

Imagen fotografía María Isabel Jasbón

trabajo o en lugar del trabajo (para las desempleadas) prevalece el rol tradicional para mujeres de asumir responsabilidades familiares en momentos de crisis, el cual las obligaba a ausentarse de clases, por consiguiente retrasarse y no acreditar las asignaturas.

También es importante destacar la proporción de los que no trabajan $(27,5 \%$ ) y que sin embargo, poseen estatus en alguno de los artículos por motivo de reprobación. En esta condición se encuentran en el artículo 33 el 54.3\%, en el artículo 34 el $40.93 \%$. En el 35 solo es el $4.69 \%$. En una de las preguntas de la encuesta, manifiestan que les hace falta una beca o apoyo económico.

Con relación al nivel educativo de los padres de los estudiantes, pareciera que el capital cultural no es la razón principal de la reprobación, porque los datos muestran que la mayoría de ellos tienen estudios de licenciatura, sin embargo hay que tomar en cuenta la situación económica por la que atraviesa el país a partir de las condiciones generadas por el modelo económico neoliberal que -entre otras cosasha disminuido considerablemente el empleo e ingreso de los profesionistas universitarios y su calidad de vida, lo que obliga a los jóvenes a estudiar y trabajar para aportar al ingreso familiar o sufragar sus gastos personales.

Como pudo observarse en los párrafos anteriores, hay ocasiones en que las mujeres parecen tener más ventajas, en otras los hombres muestran más facilidades y en otras la situación es similar para ambos. Consideramos como probabilidad que aunque prevalezca la desigualdad educativa en la población universitaria, haya situaciones en las que no existan diferencias significativas entre los reprobados de diferente sexo, puede ser también una señal de igualdad de condiciones durante la formación profesional.

\section{Opinión de los estudiantes acerca de los motivos que los han llevado a la} reprobación

\section{a) Motivos de origen psicológico}

Entre estos motivos casi la mitad de los participantes de los tres artículos (48.3\%) no le atribuye significación como causal reprobatoria. Sin embargo entre quienes identifican este factor como importante, las mujeres arrojan la mayor cantidad de casos. Destacan las respuestas que señalan la falta de motivación en un $21.58 \%$, dividido en $13.4 \%$ para las jóvenes y $8.1 \%$ para los varones; en las dificultades de atención o concentración se mencionan con un porcentaje del $10.33 \%$, distribuidos en $5.8 \%$ para el sexo masculino y $4.53 \%$ para el femenino; los problemas de depresión representan el $14.6 \%$ para los hombres y $25.4 \%$ para mujeres; la vocación por la carrera el $6.45 \%$ entre ambos y en problemas existenciales predominó el lado masculino con un 4.24\%; de ellos una persona manifiesta haber intentado suicidarse.

En este tipo de dificultades de los alumnos que viven problemas de reprobación en las licenciaturas del CUCEA, es probable que la no manifestación de las emociones esté presente en las respuestas porque todavía predominan los modelos de masculinidad en los jóvenes. En las chicas está socialmente permitido darse el lujo de bajarse del carro de la racionalidad, sin embargo también en este grupo hay quienes no los consideran causales, ya sea porque efectivamente no están presentes, como puede 
ser en los casos de artículo 33 o porque no los reconocen. De todas formas, todos, hombres y mujeres por igual, en el mismo cuestionario cuando se les pregunta cómo se sienten por haber reprobado, manifiestan su desconcierto cuando no acreditan las materias y el impacto emocional que esto tiene: depresión, desmotivación, decae su autoestima, sensación de fracaso, preocupación por su futuro y no sentirse con las aptitudes para triunfar, entre otros.

\section{b) Motivos de reprobación atribuibles al estu- diante}

En lo relativo al aprendizaje o aprovechamiento escolar, destacan principalmente las dificultades para entender la materia, 25.5\%; y los problemas relativos a hábitos de estudio con $25 \%$. En ambos casos las proporciones son parecidas en ambos sexos.

\section{c) Motivos de reprobación relacionados con los docentes}

En general, el $60 \%$ de los estudiantes consideran que existen motivos de reprobación relacionados con los profesores y el $40 \%$ no atribuye importancia a estos aspectos.

Los docentes constituyen una de las partes esenciales en el proceso de aprendizaje $y$, por lo tanto, no pueden ser excluidos de los motivos involucrados en la reprobación. Al respecto la opinión de los estudiantes señala en primer lugar las dificultades en la relación personal con los profesores en una proporción de $24.16 \%$, corresponde el $16 \%$ a los varones y el $8.16 \%$ a las jóvenes; en el resto de motivos no hay diferencias significativas entre los encuestados. Las deficiencias pedagógicas en segundo lugar (16.97\%), los criterios de evaluación (11.07\%) y el conocimiento insuficiente de la materia (7.74\%). En general, casi $60 \%$ de los alumnos considera que existen motivos de reprobación relacionados con los profesores, pero especialmente es la población masculina la que acusa mayores problemas de relaciones interpersonales con ellos.
Que el 60\% atribuya parte de su fracaso académico a causas originadas por los docentes, indica un serio problema de comunicación entre las partes, especialmente por parte de los jóvenes, quienes duplican esta situación respecto a sus compañeras. A decir de Habermas (citado por Sáenz) no hay acción comunicativa que favorezca superar las relaciones asimétricas, en este caso profesor-alumno, para tomar decisiones sobre los derechos y deberes de los sujetos involucrados en la relación.

La asimetría se expresa, por una parte, cuando se otorga la responsabilidad de la aprobación de una asignatura, al alumno, y poco se cuestiona a nivel institucional el desempeño docente. Por la otra, porque en el resultado de las calificaciones aprobatorias o reprobatorias, la decisión de los profesores gira más en torno a pretensiones de poder que de validez; esto puede atribuirse a que en las instituciones educativas la educación no es crítica, entendido por crítica"el esfuerzo intelectual y en definitiva práctico por no aceptar sin reflexión y por simple hábito las ideas, los modos de actuar y las relaciones sociales dominantes; (Horkheimer, citado por Sáenz). Esta situación es predominante en las materias de corte cuantitativo, que coincidentemente es en las que se ubica el mayor porcentaje de asignaturas que conducen a la reprobación estudiantil.

Aterrizada esta situación a la perspectiva de género, no es casual que sean los varones los que manifiesten mayor problema en las relaciones interpersonales con los profesores, porque en general tienden a reclamar calificaciones más que las mujeres, posiblemente por eso tienen menos dificultades de tipo interpersonal; por un lado la mayoría del profesorado del CUCEA son hombres y además mayores, por otro lado, las jóvenes viven en una sociedad predominantemente machista donde los comportamientos de "superioridad masculina" reforzados por la jerarquía del profesor sobre el alumno, se impone. Cabe aclarar que de esta situación no se eximen las profesoras, aunque sean minoría 
en la planta docente. En este sentido coincidimos con Juan Carlos Ramírez (2008:12) cuando afirma que "el problema no son los hombres ni las mujeres. El problema reside en los modelos patriarcales de masculinidad, en las representaciones sociales que avalan y refuerzan estos modelos, así como en las políticas públicas y en el orden social que favorece las desigualdades".

\section{Motivos relacionados con la Institución (CUCEA. U de G)}

En este apartado se presenta a grandes rasgos la valoración realizada por los estudiantes acerca del papel que desempeña la Institución ante los problemas de reprobación que los conduce al estatus irregular por los artículos 33 y 34, y a baja de la carrera en el caso del artículo 35.

En términos generales la valoración de los estudiantes acerca de los servicios que reciben de la institución se consideran deficientes y en orden de importancia se presentan los siguientes aspectos: la Información acerca de los servicios de tutoría/asesoría y del contenido de los artículos 33, 34 y 35, la atención del personal de la Coordinación de Control Escolar, las tutorías y asesorías, la oferta de grupos por materia y la atención de las coordinaciones de carrera, entre los principales.

Es importante atender aspectos tan simples como el relativo a proporcionar de forma eficaz la información respecto al reglamento vigente para aplicar los artículos. Si bien, más de la mitad reportan haberse enterado de su existencia, la información no fue suficiente para evitar o prevenir la dificultad de reprobación. En cuanto al $42.3 \%$ que no tuvo conocimiento en este particular, esta es una señal de alarma para quienes se encargan de introducir a los dicentes al mundo universitario, semanas antes de su ingreso a clases.

De manera global, la información obtenida sobre los factores de reprobación relacionados con la institución, dan cuenta de cómo se efec- túa el proceso de la reproducción social de la desigualdad en la Universidad, porque como afirma Bourdieu (1998:8-9) y se pudo constatar con los resultados obtenidos, no hay mecanismos institucionales que equiparen las condiciones de igualdad entre los estudiantes reprobados y no reprobados, o entre las diferencias de género, sino al contrario, la arbitrariedad cultural en los horarios y otros aspectos de la burocracia organizacional universitaria se refleja en ejemplos como los señalados por los entrevistados: los reprobados -hombres o mujeres- son tratados por igual y ambos tienen menos oportunidades de elegir los horarios adecuados a sus tiempos debido a que se le da prioridad a quienes acreditaron las materias $^{3}$, incluso hay algunas que ni siquiera están pensadas para los que trabajan puesto que no se ofrecen en el horario nocturno. Se beca a quienes obtienen mayores promedios y no se otorga apoyo económico a aquellos que se encuentran en situación de desventaja y, por ende, vulnerabilidad.

Como estos hay gran cantidad de ejemplos, que muestran el "darwinismo social" mencionado por Flecha (1994:59) cuando los desarrollos curriculares seleccionan a los "mejores" para darles prioridad respecto a los que tienen problemas escolares. También muestran que las condiciones ofrecidas por la Universidad son mecanismos de exclusión social en la fase de permanencia y egreso de la institución, aunado con una gran dosis de violencia simbólica. Son parte de los "aspectos encubiertos en los conceptos, relaciones, distribución de responsabilidades, que vienen dados en el funcionamiento cotidiano" de las organizaciones educativas,

3 La Universidad de Guadalajara en el nivel de educación superior (pregrado) se rige por el sistema de créditos en el que los estudiantes seleccionan las materias que cursarán en el ciclo escolar, esto incluye seleccionar el horario en el que lo harán de acuerdo a la oferta que hace la Institución de las mismas. El orden para realizar esta selección se da de acuerdo al promedio de calificaciones del ciclo escolar inmediato anterior. Los estudiantes con mejores promedios son los primeros en seleccionar materias y horario de acuerdo a su conveniencia siendo los estudiantes en condición de reprobación los últimos en seleccionarlas. Esta condición lleva a estos últimos a seleccionar materias que aún cuenten con cupo pero que el horario no les favorece. 
como afirma Sáenz del Castillo desde la Teoría Crítica de la Escuela de Frankfurt aplicada a la educación.

Por el momento el reto a enfrentar es: ¿Cómo en las condiciones de desigualdad y reproducción de la desigualdad, es posible formular y aplicar las políticas institucionales que permitan a las y los estudiantes resolver la problemática de la reprobación y repitencia?

\section{Reflexiones finales}

El presente trabajo permitió mostrar que un hecho educativo como la reprobación, refleja un problema social generado por la desigualdad. Se pudo constatar que los jóvenes en situación de artículos 33, 34 y 35, tuvieron la oportunidad de ingresar a la universidad después de un proceso de selección altamente competitivo, sin embargo, ya como estudiantes regulares fue primordialmente la necesidad de trabajar debido a su situación socioeconómica, aunada a condiciones institucionales, la causa principal de su bajo rendimiento académico.

En el contexto socioeconómico actual marcado por la globalización y la crisis de las políticas neoliberales, el trabajo precario en el ámbito profesional y el desempleo que padecen especialmente las y los jóvenes que poseen educación superior, reflejan un panorama desalentador y crítico al que se suma el peso cultural que impone valores tradicionales propios del modelo patriarcal que dificultan el empoderamiento de las mujeres universitarias, especialmente en el campo laboral. Más desalentador todavía es el futuro para quienes se circunscriben en la situación académica de reprobación, especialmente cuando los cambios educativos en la institución, lejos de alejarse de la lógica neoliberal y acercarse al entendimiento de las dinámicas que se generan a partir de las interacciones entre los diferentes actores, como proponen McLaren y Huerta (2010) la refuerza.
Afortunadamente, a pesar de los obstáculos para el cambio social surgen voces a favor de la transformación social y a favor de la emancipación y equidad de género. Para la primera, clásicos como McLaren (2010), desde la pedagogía crítica y la pedagogía de frontera abren caminos para transitar a cambios educativos incluyentes; para la segunda, surgen alternativas: desde la teoría feminista latinoamericana, la propuesta "ética del cuidado" como responsabilidad social que exige oír la voz del otro (Carosio, 2009, p 249), o desde las masculinidades, promover modelos de masculinidad que incluyan el respeto y reconocimiento por los afectos y las emociones (Hockman, 2008, p. 14). En este sentido y ante la problemática presentada en este artículo, escuchar y respetar las condiciones, opiniones y sentimientos de los dicentes debe ser un compromiso institucional para resolver las desventajas de la población universitaria más vulnerable.

Proponemos una política efectiva de equidad social y de género en la Universidad que oriente y del seguimiento constante a los programas educativos a fin de contribuir a disminuir la brecha de la desigualdad social y mejorar las condiciones de estudio del alumnado que presenta dificultades económicas, psicológicas y/o académicas, con especial atención a la población reprobada. Proponemos también impulsar valores que fomenten un nuevo orden con valores como el deber y responsabilidad para con los demás o la justicia social, que desgraciadamente no siempre son considerados dentro de las prácticas cotidianas.

Empezar con medidas de emergencia para ofrecer apoyo efectivo de atención, información y asesorías especializadas por asignatura, instauración de cursos en línea y presenciales con horarios adecuados para asegurar la inscripción de los estudiantes que trabajan y se encuentran en situación de los artículos 33, 34 y 35. Continuar con reformas educativas tendientes a minimizar y acabar con los factores de exclusión y marginación que dan lugar a la desigualdad educativa. 


\section{Referencias Bibliográficas}

Bourdieu, P. y Passeron, J. (1998). La Reproducción. Elementos para una teoría del sistema de enseñanza. México. Ed. Laia S.A. Distribuciones Fontamara.

Carosio, Alba (2009). “Feminismo latinoamericano: imperativo para la emancipación". En Girón Alicia (coord.). Género y Globalización. Buenos Aires. CLACSO.

Flecha R. (1994). Las nuevas desigualdades educativas. En Castells M. (comp.) Nuevas perspectivas críticas en educación. España. Ed. Paidós Ibérica.

Hockman, A. (2008). Prólogo. En Ramírez Rodríguez, Juan Carlos y Uribe Vazquez Griselda (coords.). Masculinidades, el juego de género de los hombres en el que participan las mujeres. México. Plaza y Valdez editores, Universidad de Guadalajara, PIEGE, UNFPA, Academia Jalisciense de Ciencias A.C.

Mc Laren P y Huerta L (2010). El cambio educativo, el capitalismo global y la pedagogía crítica revolucionaria. En Revista Mexicana de Investigación Educativa, Vol XV (47), p. 1124-1130. México.

Núñez Noriega, G. (2008). "Los 'hombres' en los estudios de género de los 'hombres': un reto desde los estudios queer". En Ramírez Rodríguez, Juan Carlos y Uribe Vázquez Griselda (coords.). Masculinidades, el juego de género de los hombres en el que participan las mujeres. México. Plaza y Valdez editores, Universidad de Guadalajara, PIEGE, UNFPA, Academia Jalisciense de Ciencias A.C.

Reglamento General de Promoción y Evaluación de Estudiantes de la Universidad de Guadalajara (2000). Universidad de Guadalajara. Disponible en: www. udg.mx

Ramírez Rodríguez, J. C. (2008). “El género de los hombres: un subcampos de estudios en expansión" (Nota introductoria). En Ramírez Rodríguez, Juan Carlos y Uribe Vázquez Griselda (coords.). Masculinidades, el juego de género de los hombres en el que participan las mujeres. México. Plaza y Valdez editores, Universidad de Guadalajara, PIEGE, UNFPA, Academia Jalisciense de Ciencias A.C.
Ramos Escandón, C. (1992). “El género en perspectiva: de la dominación universal a la representación múltiple" (Nota introductoria). Ed. Universidad Autónoma Metropolitana. Unidad Iztapalapa. México.

Ruiz, R. y Rodríguez, G. (coords), (2005). “Feminización de la matrícula en educación superior en América Latina y el Caribe". México. Universidades, Julio-diciembre No. 030 y Unión de Universidades de América Latina -UDUAL-, D.F. México. Disponible en http://redalyc. uaemex.mx/pdf/373/37303005.pdf

Sáenz del Castillo A. (s/f). "Teoría Crítica y Educación". Disponible en http://educritica.ideoneos.com

Universidad de Guadalajara (2000). “Reglamento General de Evaluación y Promoción de Estudiantes de la Universidad de Guadalajara". Disponible en: www.udg. $\mathrm{mx}$.

Universidad de Guadalajara. (2009). "Sistema Institucional de Información y Administración Universitaria (SIIAU)". Disponible en: www.udg.mx

Universidad de Guadalajara. "Informe de actividades 2010-2011 del rector del CUCEA, Mtro. Itzcoatl Tonatiuh Bravo Padilla". Disponible en http://www.udg. $\mathrm{mx} /$ node/15433.

Universidad de Guadalajara. "Informe de actividades 2011-2012 del rector del CUCEA, Mtro. Itzcoatl Tonatiuh Bravo Padilla". Disponible en: www.udg.mx

Viveros, M. (2008). “Teorías feministas y estudios sobre varones y masculinidades. Dilemas y desafíos recientes". En Ramírez Rodríguez, Juan Carlos y Uribe Vazquez Griselda (coords.). Masculinidades, el juego de género de los hombres en el que participan las mujeres. México. Plaza y Valdez editores, Universidad de Guadalajara, PIEGE, UNFPA, Academia Jalisciense de Ciencias A.C.

\section{Cómo citar este artículo:}

Silva, Rodríguez, Vicente, Ruiz. (2013). Reflexiones en torno a la reprobación estudiantil, desigualdad social y equidad de género en estudiantes de licenciatura. Espiral, Revista de Docencia e Investigación. 2 (2), 79-90 